\title{
Self assembling proteins as matrix for the construction of optical devices
}

\author{
U. Weinert, T. Günther, J. Raff \& K. Pollmann \\ Forschungszentrum Dresden-Rossendorf e.V., Germany
}

\begin{abstract}
Self-assembling biomolecules are widespread in nature and attractive for technical purposes due to their size and highly ordered structures in nanometer range. Surface-layer (S-layer) proteins are one of those self-assembling molecules and their chemical and structural properties make them quite attractive for nanotechnical purposes. They possess a high content of functional groups so a sequential coupling of functional devices is possible and their ability to self assemble in aqueous solutions or on surfaces, e.g. $\mathrm{SiO}_{2}$ wafers, qualifies them for nanotechnical applications. In this work, first experiments were done in order to construct a sensory device containing S-layer proteins as matrix to bind optical elements and analytes for detection of specific substances. The S-layer proteins were isolated from the Lysinibacillus sphaericus strain JG-A12 recovered from a uranium mining waste pile in Germany. As optical elements fluorescent dyes or quantum dots can be used. Three different fluorescent dyes which are able to perform a Fluorescence resonance energy transfer (FRET) were used and coupled to the S-layer proteins. As receptor molecule aptamers were chosen due to their high specifity and stability towards many chemicals. Aptamers are short oligonucleotides which are able to bind specific molecules via their three dimensional structure. In this work, a model aptamer was used that is specific towards human thrombin. The aim was to construct a sensor system which is able to detect specific substances in very low concentration ranges in aqueous solutions.
\end{abstract}

Keywords: S-layer proteins, fluorescent dyes, aptamers. 


\section{Introduction}

S-layer proteins represent the outermost cell envelop compound of numerous bacteria and almost all archaea. These structure proteins feature a lot of functions such as protection, shape and molecular sieves. They are able to self assemble in aqueous solutions and on surfaces and form regular sheets exhibiting a paracrystalline nanostructure [1]. Generally, S-layer proteins provide a huge amount of orientated functional groups that can be used for coupling of molecules to the surface, thus introducing a high level of functionality in a small device. The possibility to create highly regular arrays of nanostructured multifunctionalized surfaces, makes S-layer proteins highly attractive for the construction of sensory layers.

Here we present a new concept of biosensors based on the application of S-layers. This biosensor will be able to detect various substances in a very low concentration range, e g. pharmaceuticals in clarified waste water. Figure 4 shows the functionality of the biosensor with following compounds:

(1) S-layers that are used for the nano-structuring and functionalization of surfaces such as $\mathrm{SiO}_{2}$-wafers or glass.

(2) Aptamers that are coupled to S-layers and work as receptors. Aptamers are oligonucleotides that specifically bind chemical compounds via their threedimensional structure. In the present study, aptamers that specifically bind thrombin were used as a model.

(3) Two different fluorescence dyes with overlapping excitation and emission spectra. A unit cell of the S-layers has a size of $12.8 \mathrm{~nm}$ and comprises four protein monomers. Therefore coupled fluorescence dyes are in a close proximity, thus favouring a fluorescent resonance energy transfer (FRET). FRET means a radiation free energy transfer from one fluorescence dye (donor) to another fluorescence dye (acceptor). The energy transfer causes a decrease in donor fluorescence and an increase in acceptor fluorescence. Such a transfer system is very sensitive against environmental changes and can be easily disturbed e.g. by the binding of analytes to the aptamer.

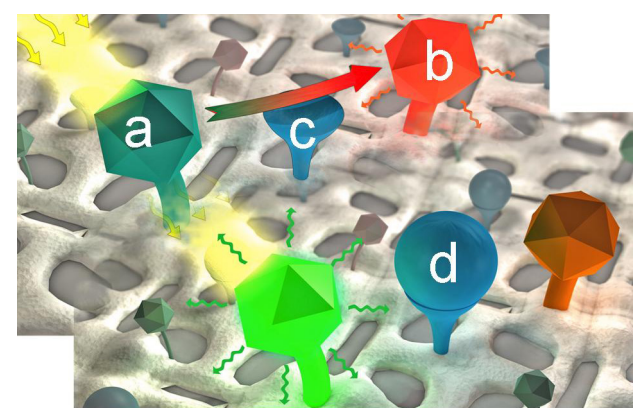

Figure 1: Schematic drawing of a sensory device containing two fluorescent dyes ( $a$ and $b$ ) which are able to perform a FRET and one aptamer (c) specific for one analyte (d). 
The S-layer proteins of L. sphaericus JG-A12 that were used for the present study contain $10 \mathrm{~mol} \% \mathrm{NH}_{2}$ and $12 \mathrm{~mol} \% \mathrm{COOH}$ groups, consequently providing many residues for functionalization. These groups of the S-layers can be easily coupled with fluorescent dyes by using crosslinking reagents like 1-Ethyl-3-(3-dimethylaminopropyl)carbodiimid (EDC). Additionally, hydroxylgroups of the protein can be coupled with aptamers. In the present study the coupling of the three different fluorescent dyes Hilyte488, Hilyte555 and TAMRA and the anti-thrombin aptamer was performed and the results analyzed.

\section{Materials and methods}

\subsection{Isolation of S-layer proteins}

The isolate L. sphaericus JG-A12 was grown in Nutrient Broth medium (6 g/l, Mast Group Ltd., Merseyside, UK) in a $50 \mathrm{~L}$ bioreactor over night at $\mathrm{pH} 7$ and $30^{\circ} \mathrm{C}$. Extraction of S-layer proteins was performed according to [2]. Determination of protein concentration was determined by Lowry [3] and purity of S-layer proteins was confirmed by SDS-PAGE. Proteins were stored in standard buffer solution at $\mathrm{pH} 7.5$ containing $50 \mathrm{mM}$ Tris, $2 \mathrm{mM} \mathrm{MgCl} 2$ and $3 \mathrm{mM} \mathrm{NaN}_{3}$.

\subsection{Functionalization of S-layer proteins}

\subsubsection{Binding fluorescent dyes to S-layer proteins}

S-layer sheets were centrifuged and resolved in $50 \mathrm{mM}$ phosphate buffer at $\mathrm{pH} 5.6$ to give a final concentration of $10 \mathrm{mg} / \mathrm{mL} \mathrm{S}$-layer protein polymer solution. Coupling experiments were performed with three different fluorescence dyes. As donor molecule, Hilyte 488 was chosen and used as hydrazide. As acceptor molecules Hilyte555 and TAMRA were tested and used as hydrazide or succimidyl ester (SE), respectively. The carboxyl groups of the S-layer proteins were activated with EDC in a final concentration of $20 \mathrm{mM}$. The reaction started immediately. Reaction tubes were incubated overnight at $4^{\circ} \mathrm{C}$ in dark. Afterwards the samples were centrifuged and washed several times to remove remaining fluorescent dyes. The coupling of fluorescent dyes to the proteins was quantified by UV/VIS (Ultrospec 1000, Amersham Pharmacia Biotech, USA). For theses measurements $50 \mu \mathrm{L}$ of the protein solutions were dissolved in $50 \mu \mathrm{L}$ of $6 \mathrm{M}$ guanidine solution $\mathrm{pH} 7$ and incubated for one hour in order to get a monomer solution.

\subsubsection{Binding anti-thrombin-aptamer to S-layer proteins}

Oligonucleotides with the sequence GGTTGGTGTGGTT [4], a TAMRA modification at its $3^{\prime}$ end and a SH-group at its $5^{\prime}$ end were used as antithrombin-aptamer. The aptamer was linked to $\mathrm{OH}$-groups of the S-layer protein via its $\mathrm{SH}$-group by using $\mathrm{N}$-[p-Maleimidophenyl]isocyanate (PMPI) as crosslinking reagent. The reaction was started by the addition of PMPI, dissolved in DMSO, to a solution of S-layer proteins and aptamers, dissolved in distilled water at $\mathrm{pH} 7$ with a final molar ratio of 1:2.5:500 (protein:aptamer:PMPI). 
Reaction was performed overnight at $4{ }^{\circ} \mathrm{C}$. Precipitated PMPI was removed by centrifugation and selective resuspension. After reaction, the residual aptamers in the supernatant were removed. The binding of aptamer to S-layer proteins was controlled via UV/VIS spectroscopy and quantification of linked aptamer was done by measuring the uncoupled aptamer in the supernatant.

\section{Results and discussion}

\subsection{Binding fluorescence dyes to S-layer proteins of $L$. sphaericus JG-A12}

In a first step, UV/VIS spectroscopy was used to demonstrate that the S-layer protein of $L$. sphaericus JG-A12 can be successfully modified with each of the three fluorescent dyes. TAMRA was linked to the $\mathrm{NH}_{2}$-groups whereas Hilyte488 and Hilyte555 were linked to the COOH-groups. As an example, the results on S-layer modifications with Hilyte 488 or TAMRA and with both dyes together are shown in figure 2. Unfortunately, the spectra of free and protein bound dyes show a slight peakshift and an overlap of donor and acceptor absorption. These facts make it difficult to quantify the total amount of bound fluorescence dyes by UV/VIS spectroscopy. Therefore fluorescence spectroscopy was used to determine the amount of bound fluorescence dyes. If two fluorescence dyes are linked to the protein the total amount of each fluorescence dye can be determined and compared with samples where only one fluorescence dye is linked.

For fluorescence measurements the optimal excitation and emission wavelength of the chosen fluorescence dyes in this experimental setup have to be determined. In result Hilyte 488 was excited at a wavelength of $500 \mathrm{~nm}$, TAMRA at a wavelength of $556 \mathrm{~nm}$ and Hilyte555 at a wavelength of $553 \mathrm{~nm}$. Fluorescence spectra of protein modified with TAMRA and protein modified with a combination of Hilyte 488 and TAMRA show same emission intensities at $580 \mathrm{~nm}$ when excited at a wavelength of $556 \mathrm{~nm}$ (see figure 3). This fact indicates that comparable amounts of TAMRA were linked to the protein in both samples. An equal approach to determine the amounts of linked Hilyte 488 by excitation with light of a wavelength of $500 \mathrm{~nm}$ is not possible, as an occurring FRET will cause a decrease of the fluorescence intensity at $530 \mathrm{~nm}$. This effect can be seen in figure 3, whereas the fluorescence intensity at $580 \mathrm{~nm}$ simultaneously increases in comparison to the emission spectra of protein modified with TAMRA and excited at a wavelength of $500 \mathrm{~nm}$. Both observations together are a strong indication for a FRET event.

A modification of S-layer protein with Hilyte 488 and Hilyte 555 at the same time reduces the total amount of bound molecules for both dyes in comparison to samples with only one linked fluorescence dye (data not shown). This can be easily explained by the binding of both dyes via $\mathrm{COOH}$ groups so that less binding sites are available for each fluorescence dye. Furthermore an excitation of Hilyte 555 at a wavelength of $500 \mathrm{~nm}$ shows a clear emission at $565 \mathrm{~nm}$ which 


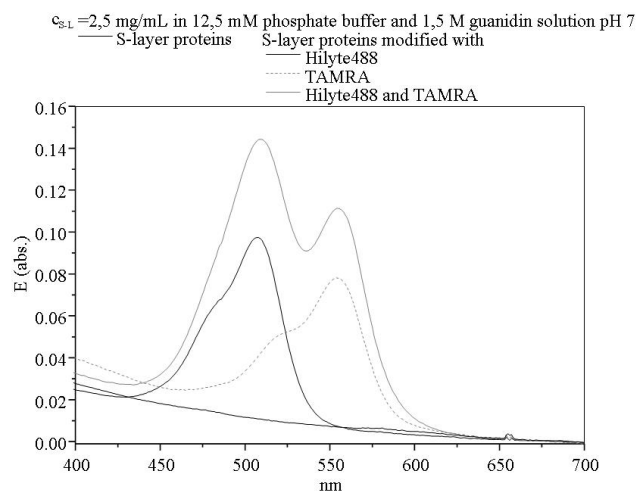

Figure 2: $\quad$ UV/VIS spectra of S-layer proteins (solid black line) modified with Hilyte488 (dashed line), with TAMRA (dotted line) and with both fluorescence dyes (solid grey line).

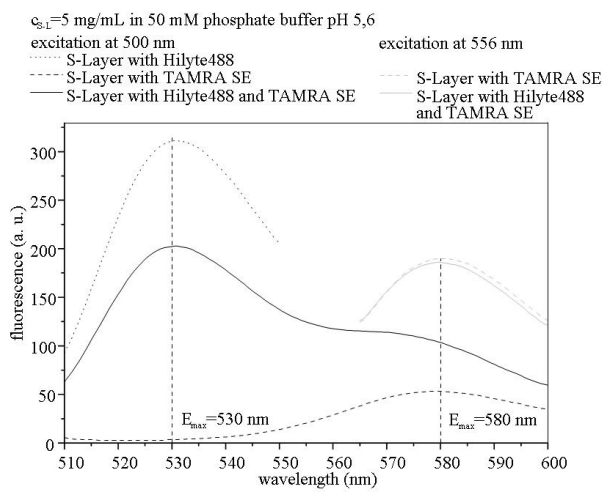

Figure 3: Fluorescence spectra of S-layer proteins modified with Hilyte488, TAMRA or with both fluorescence dyes; excitation wavelength for Hilyte488 was $500 \mathrm{~nm}$ and for TAMRA $556 \mathrm{~nm}$.

hinders a potential FRET detection due to the fact that a donor excitation simultaneously excites the acceptor. Therefore further experiments will be done with the Hilyte488 and TAMRA as potential FRET pair.

\subsection{Binding anti-thrombin-aptamers to S-layer proteins of $L$. sphaericus JG-A12}

As presented in figures 4 and 5, anti-thrombin-aptamers were successfully coupled to S-layer proteins of L. sphaericus JG-A12 via PMPI. Modification degree was determined by measuring the absorption maxima of uncoupled antithrombin-aptamer that remained in the supernatant after crosslinking. Whereas light microscopic images show that all S-layer polymers are modified with 
aptamers, the results of UV/VIS spectroscopy demonstrate that only $20 \%$ of the applied S-layer protein monomers were modified with aptamers. Future work will focus on an enhanced modification rate in order to increase the sensitivity of the finally assembled sensor. Additionally S-layer proteins will be stabilized with EDC in order to inhibit degradation after the coupling of aptamer.

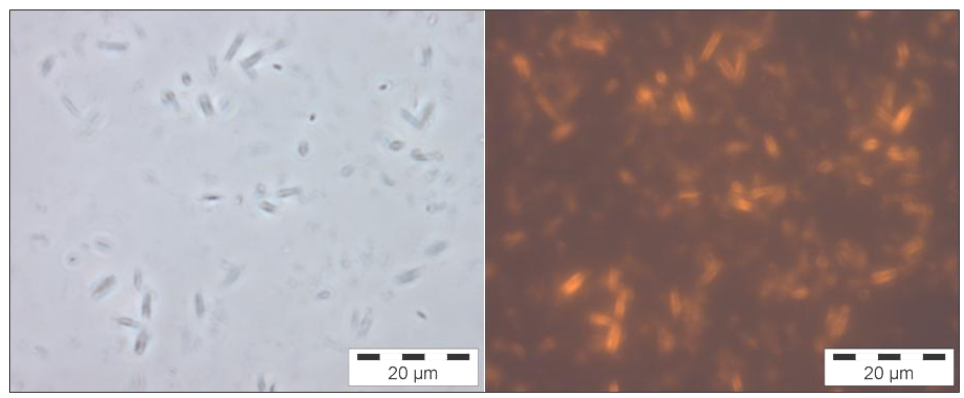

Figure 4: Light microscopic image of S-layer proteins modified with antithrombin-aptamer in phase contrast mode and in fluorescence mode using a filter between 540 and $550 \mathrm{~nm}$.

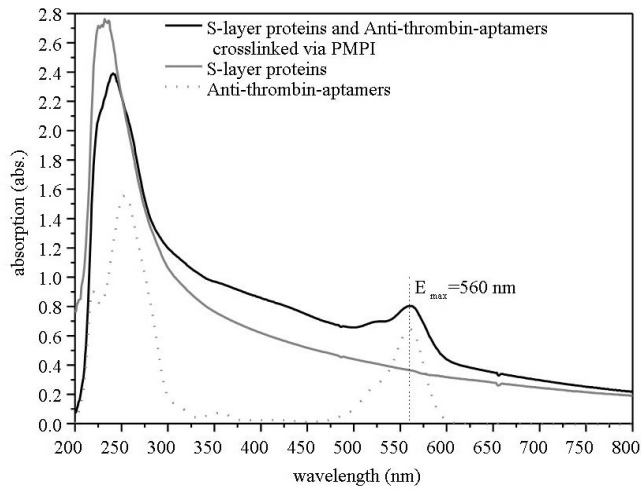

Figure 5: UV/VIS spectra of unmodified S-layer proteins (grey line), free anti-thrombin-aptamer (dotted line) and S-layer proteins modified with anti-thrombin-aptamer (black line).

\section{Outlook}

For the construction of a biosensor that is composed of S-layers, fluorescence dyes and aptamers, important steps are the stable coupling of the functional molecules to the S-layer proteins without loosing the functionality of those molecules and the structure of the S-layers. The presented results demonstrate that such a functionalization is possible while keeping the structure of the S-layer polymers. In further steps affinity studies of the coupled anti-thrombin-aptamers 
will be done in order to verify the intactness of the molecules. In addition, lifetime measurements will be performed in order to prove the occurrence of an energy transfer between the fluorescence molecules.

\section{Acknowledgement}

This study was kindly supported by the German Federal Ministry of Education and Research $(B M B F)$, grant.01RB0805A.

\section{References}

[1] Sará, M. and Sleytr, U. B., S-layer proteins, Journal of Bacteriology, 182(4), pp. 859-868, 2000

[2] Raff, J. et al. Chem Mater 15(1), pp. 240-244, 2003

[3] Lowry, O. H., Rosebrough, N. J., Farr, A. L., Randall, R. J., Protein measurement with the folin phenol reagent. Journal of Biological Chemistry, 193(1), pp. 265-275, 1951

[4] Bock, L. C., Griffin, L. C., Latham, J. A., Vermaas, E. H., Toole, J. J., Selection of Single-Stranded-DNA Molecules That Bind and Inhibit Human Thrombin. Nature, 355(6360), pp. 564-566, 1992 